Proceedings of the Operational Research Society Simulation Workshop 2021 (SW21)

M. Fakhimi, D. Robertson, and T. Boness, eds.

DOI: https://doi.org/10.36819/SW21.039

\title{
ONTOLOGY DERIVED CONCEPTUAL MODELING FOR SIMULATION
}

\author{
Nurul Saleh \\ Brunel University London \\ London, United Kingdom \\ nurul.mdsaleh@brunel.ac.uk
}

\author{
David Bell \\ Brunel University London \\ London, United Kingdom \\ david.bell@brunel.ac.uk
}

\begin{abstract}
An ontology is one of the way to represent domain knowledge into a human-understandable and machinereadable format. Meanwhile, an ontology in simulation has been used as a conceptual model to explicitly describes the modelers' perspective of the domain. This study proposes a rigorous method that systematically extracts domain concepts, synthesizes processes within the domain and build an ontology for simulation modelling - a Minimal-Viable Simulation Ontology (MVSimO). MVSimO can be viewed as a derived conceptual model that supports modelling and simulation through abstraction and simplification of the domain. The novel approach presented curates the modelers' perspective of the real-world by extracting concepts from existing knowledge and synthesizes the processes involved (demonstrated in A\&E departments). The effectiveness of this method is reviewed by comparing MVSimO ontological model to the existing model. Evaluation results are encouraging, providing possibilities to improve an ontology for simulation when access to experts is limited.
\end{abstract}

\section{Keywords:}

Ontology, Conceptual Modeling, Simulation Modeling, Formal Concept Analysis

\section{INTRODUCTION}

The Semantic Web relies heavily on the underlying data structure for the purpose of comprehensive and transportable machine understanding. The term "Semantic Web" refers to the Web of linked data and the technologies enable creation of data stores on the Web, build vocabularies, and rules for handling data. Linked data of Semantic Web stack are empowered by technologies such as RDF, SPARQL, OWL and SKOS. Therefore, the success of Semantic Web depends strongly on the proliferation of ontologies, which facilitates knowledge acquisition by ontology engineers with the help of domain expert.

To build an ontology-based application, a conceptual model used in application development is typically supported by domain experts to gather domain requirements (Robinson 2013). For example, in developing a simulation, conceptual modeling represents a composition of concepts which help to view the abstraction of the real world system. Meanwhile, the modeler or researcher attain the optimal knowledge of the domain with the semantic representation of ontology, are able to improve the translation of real-world knowledge to model representation. This increase the chances of getting the correct simplification of the domain by making the domain assumption explicit (Noy, McGuinness, et al. 2001). Although ontologies have been introduced as conceptual model for a semantically-defined application, the development of the new ontology can be tedious and costly (Lonsdale, Embley, Ding, Xu, and Hepp 2010, Simperl 2009). The research presented in this paper is a development of a Minimal Viable Simulation Ontology (MVSimO) as an ontology derived conceptual model for simulation modeling.

For a complex domain, the observed phenomena generate the understanding and help the modeler in the abstraction and simplification process of the real-world. These processes underpin the work in this 


\section{Saleh and Bell}

paper by extracting the important elements and concepts of the A\&E departments and develop MVSimO. This paper focuses on the first phase of MVSimO development which it reuses existing ontologies from the healthcare domain that are directed toward simulation for A\&E departments. The phase where processes are synthesized using Formal Concept Analysis will not be discussed in detail. The definition of MVSimO is adopted from the definition of Minimal Viable Product by (Ries 2009): "the version of a new product which allows a team to collect the maximum amount of validated learning about customers with the least effort". Domain knowledge from real data and generic pathways is used to perceive the domain understanding of A\&E departments. The study is conducted by reusing the domain knowledge, thus saving the time and effort spent in requirement gathering and ontology design process. During the evaluation of MVSimO, an existing simulation model developed by experts is used for validation to gain an insight into the proposed work.

This study follows a design research approach which (1) identifies the problem area and its relevance from a real-world environment and previous research, (2) develops the model as a design artefact, and (3) evaluates the model through a relevant scenario. The paper is structured as follows. Section 2 discusses the background of the study, simulation in A\&E departments, ontology representation and Formal Concept Analysis (FCA) with respect to a conceptual model. Section 3 looks at the domain conceptualization for simulation modeling from the perspective of A\&E departments, and explain the detailed map of A\&E pathways. Section 4 briefly discusses the mathematical approach of domain attributes exploration using FCA. Finally, Section 5 explains the development of MVSimO and Section 6 discusses the evaluation conducted for this study and the conclusion respectively.

\section{Study Background}

\subsection{Simulation in A\&E Departments}

The emergency department, widely known as accident and emergency or A\&E department in healthcare services plays a major role to save people's lives, and more importantly to reduce death and disease rate in public (Aringhieri, Bruni, Khodaparasti, and van Essen 2017). The departments refer to the sub-system that provides medical treatment to urgent need patients, and is the most critical unit since they are one of the first unit responsible in treating life-and-death situation (Gul and Guneri 2015). Figure 1 shows the process journey in A\&E departments. The figure presented in the project "A Better A\&E" by PearsonLloyd shows the different stages from patient's check-in, assessment by staff, receiving treatment from medical staff, and the outcomes of the process.

Due to the complexity of A\&E, simulation has been used by decision makers to understand the processes and behaviours(Aringhieri, Bruni, Khodaparasti, and van Essen 2017, Traore and Zeigler 2018). Simulation models conduct a preliminary test or trial changes for a safe and efficient care deliverable implementation (Günal and Pidd 2009, Aringhieri, Bruni, Khodaparasti, and van Essen 2017) and at the same time helps in finding the optimal solution to overcome problems appear within the department (Gul and Guneri 2015). Attributes to simulate real world situations help decision makers to predict the output of a proposed solution in real-world phenomena using Discrete-event simulation (Lebcir, Demir, Ahmad, Vasilakis, and Southern 2017), System Dynamic (Pidd 2014) and Agent-based simulation (Chahal, Eldabi, and Young 2013). For the purpose of this study, DES is used due to the widespread agreement of its generality among simulation models (Guizzardi and Wagner 2010).

\subsection{Ontology and FCA}

To ensure the usefulness of simulation model in a complex and heterogeneous system like A\&E departments, the model is required to accommodate the behaviours and interaction within the domain (Isern and Moreno 2016, Baboolal, Griffiths, Knight, Nelson, Voake, and Williams 2012). Consequently, an ontology is generally assumed to play a role in setting up a common ground to provide knowledge sharing among subject domain and also describe, standardize and represent an object or instance in the domain (Grolinger, 


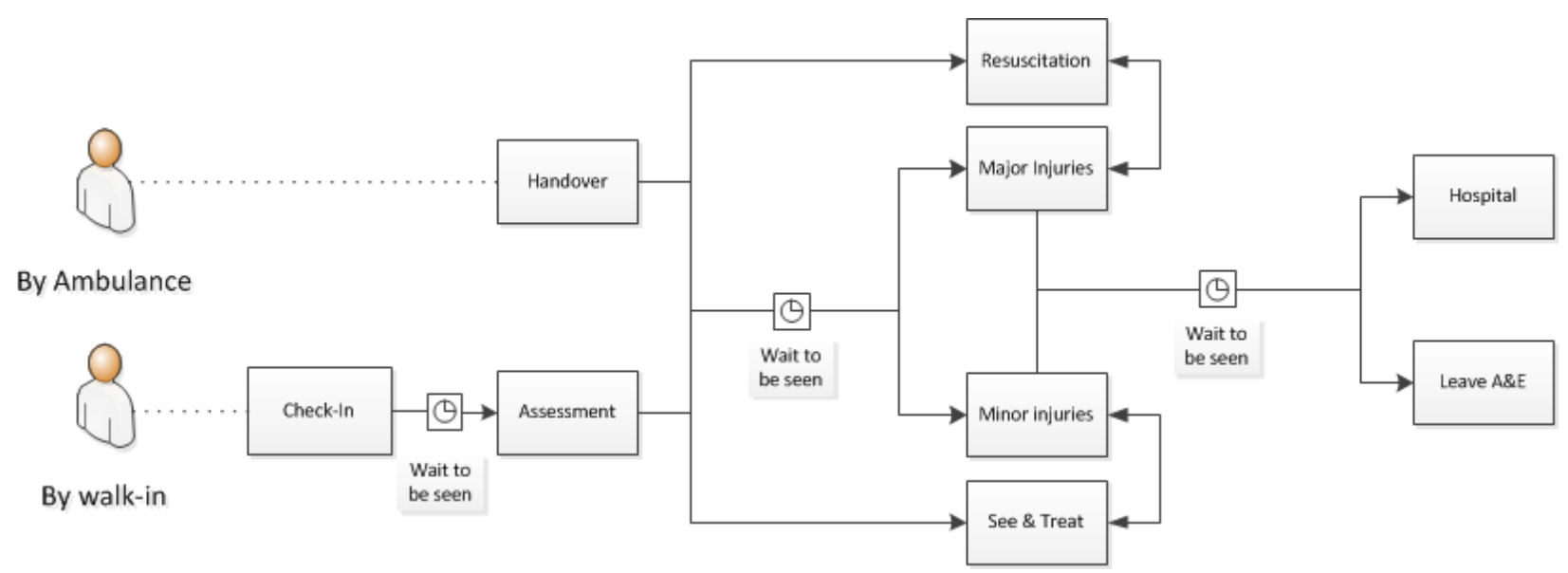

Figure 1: A\&E Process Flow

Capretz, Marti, Srivastava, Grolinger, and Capretz 2012, Huang 2016). This is because the basic foundation of ontology itself is a formal specification of conceptualization (Gruber 1993). Ontologies can accurately define a domain using classes, properties, relationships and instances hence support the determine the content of the simulation model to build the model that contains explicit detail of the domain. The novelty of the proposed approach in this paper, is that ontology is not simply build by reusing exsiting ontologies, but instead synthesize the processes performed in the departments using Formal Concept Analysis (FCA) approach.

FCA was developed in the early 1980s as a mathematical perception for concept formalization and conceptual thinking (Wille 1982). According to its philosophical definition (Wille 1982), a concept is composed of a set of objects as its extensions and a set of attributes as its intentions. Examining the process and entities in A\&E departments using FCA, gives the ability to draw new relationships that can be used to represent MVSimO class and properties. Taken together, this suggests that the aggregation of ontology and FCA during conceptual modeling may help to better understand the domain and its emerging issues, thus making the proposed model more usable and beneficial even when access to experts' opinions are limited.

\section{A\&E Case Study}

\subsection{Domain Conceptualization}

In the first phase of MVSimO development, the existing ontologies are used as to represent the domain. The requirement is defined to determine which ontology to be selected and reused to best represent the domain and the application to be modeled. Here, the domain are conceptualized to discover the candidate ontologies. Domain conceptualization phase is conducted based on general model theory by (Stachowiak 1973) - mapping, reduction and pragmatic. Mapping: To map the process in the A\&E departments, the process flow 1 is divided into modules. In the setting for simulation of A\&E departments, the modules are "healthcare" and "hospital". These modules are determined based on the intention on what to model. Reduction: The A\&E department is a large system involving several resources and heterogeneous patient type within a complex and well-organized process (Ghanes, Wargon, Jemai, Jouini, Hellmann, Thomas, and Koole 2014). Hence, ones need to identify the assumptions and ontological commitment that each module should comply to. The description of the conceptual model is the commitments outline in the specifications requirements of MVSimO. Pragmatic: In order to obtain a suitable ontology to reuse for MVSimO, in general, the ontologies should comply to the processes conducted in A\&E departments. From these three general rules, five modules are deduced: 'healthcare', 'hospital', 'emergency department', 'process' and 'patient data'. These modules are used as keywords to search for existing ontology available on the internet. 


\section{Saleh and Bell}

Generally the modules obtained from this process represent subclasses of MVSimO. Considering ontology repositories that are under active development, Bioportal ${ }^{1}$ and Ontobee ${ }^{2}$ are used to search for existing ontologies using modules-represented keyword obtained.

The search returned 31 results for BioPortal and 23 results for Ontobee. From the total of 54 ontologies minus duplicate ontologies from the same keywords, only 7 ontologies fit the criteria as outline by (Malone, Stevens, Jupp, Hancocks, Parkinson, and Brooksbank 2016). The 7 selected ontologies are:

- (HEIO) Regional Healthcare System Interoperability and Information Exchange Measurement Ontology

- (OMRSE) Ontology of Medically Related Social Entities

- (GENEPIO) The Genomic Epidemiology Ontology

- (OOSTT) Ontology of Organizational Structures of Trauma centres and Trauma systems

- (TRIAGE) Nurse Triage

- (TRANS) Nurse Transitional

- (RNPRIO) Research Network and Patient Registry Inventory Ontology

The discovery process follows the guideline developed by (Malone, Stevens, Jupp, Hancocks, Parkinson, and Brooksbank 2016), to ensure that the ontology is about a specific domain of knowledge, or in this case an appropriate amount of knowledge to cover the modules. Assumption is made that BioPortal and Swoogle are contributed by domain experts from the Semantic Web community and are under active development. From the selected ontologies, ontology merging and integration are conducted to select suitable objects, instances and properties to be reused in MVSimO. This discovery process starts from domain conceptualization that leads to modules identification and selecting candidate ontologies able to replace the role of experts in determining the abstracted and simplified simulation to be modeled.

\subsection{Space-Time-Process Map}

To map processes involve in A\&E departments to discrete-event simulation paradigm, a new detailed pathways called Space-Time-Process (STP) map is created from a generic pathways of A\&E process flow. The method of extracting the process element based on space, time and event for the ontology development has adopted the dataset analysis as proposed by (Sider et al. 2001) and (De Cesare, Juric, and Lycett 2014). To obtain the STP map, the process element based on space, time and event has adopted the dataset analysis as proposed by Sider et al. (2001) and De Cesare et al. (2014), for the ontology development method.STP map (Figure 2) is produced as a means to improve the representation of the domain by providing a new dimension to the existing process flow. The three-dimensional map illustrates the processes in the department and depicts the events in space and time dimension blocks(De Cesare, Juric, and Lycett 2014). The mapping process extracts possible process elements for the development of MVSimO model based on DES paradigm and provides the first insight from a modeler's perspective. From the map, the processes are separated into blocks of activity and entity. The blocks are lined into sequence order to resemble the flow in A\&E that have been categorised into 4 main processes: Check-In, Assessment, Treatment, and Outcome. Each process block is then labelled as process, activity and entity.

The process begins with a patient check-in, either by walking to the hospital or by using an ambulance service, patient then waits to be assessed by a nurse before proceeds to the triage assessment, receives treatment by a nurse or a medical staff, and finally waits for the outcome of whether to be discharged from the hospital or admitted to the ward. The processes take place in their designated space or location in the department within specific time. For example: A process of Check-in within $\mathrm{t}_{1}$ and $\mathrm{t}_{2}$, has an event of Patient Check-in with Receiptionist,Nurse as the entities. The process-event block represents each event that will be modeled for the discrete-event simulation. During the transition from one process to another,

\footnotetext{
${ }^{1}$ http://bioportal.bioontology.org/

${ }^{2}$ http://www.ontobee.org/
} 


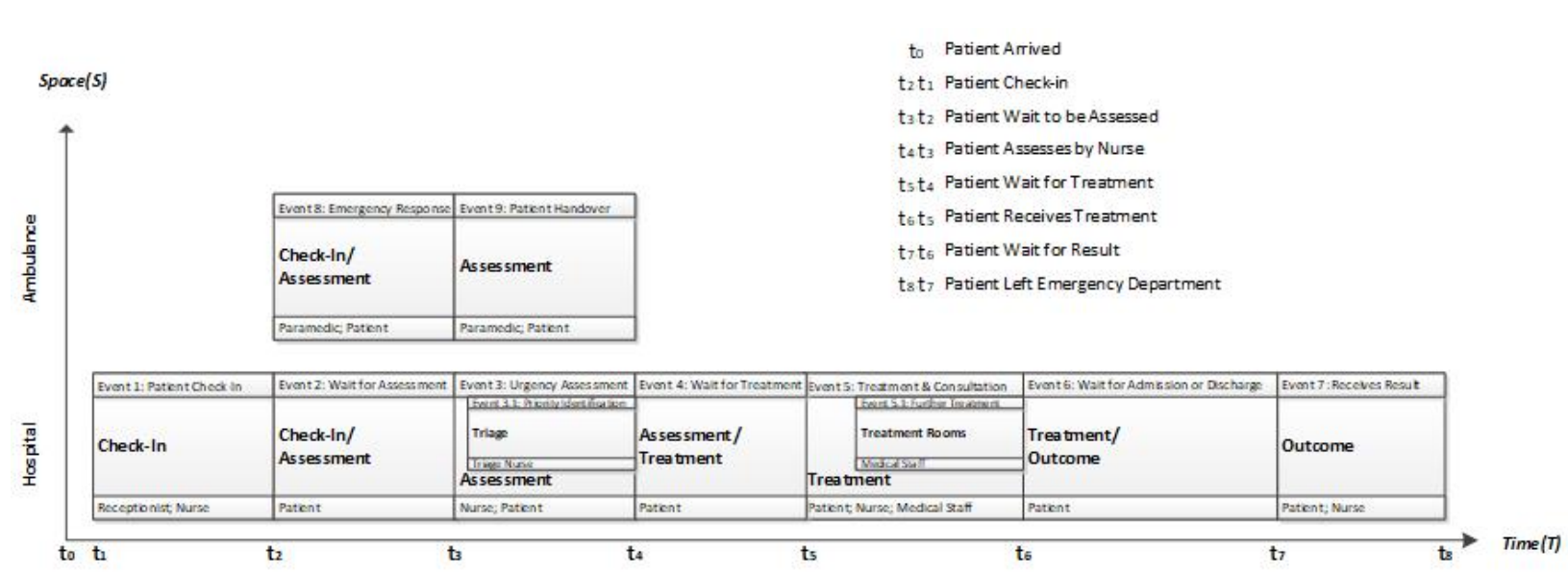

Figure 2: A Space-Time-Process of 'Patient Flow in A\&E Departments'

the patient has to wait in a queue. This suggests a delay in the process, for example between $t_{2}$ and $t_{3}$ of the process Check-In/Assessment, the event of Wait for Assessment with the entity of Patient occurs. In another example of Assessment event, there is also a sub-event which occurs in a sub-location in the department. The Triage process is conducted by a Triage Nurse to run Priority Identification.

STP map, in a three-dimensional perspective (space, time and event), help in deciding how the A\&E process elements can be extracted and modelled. Starting from here, all steps taken are based on STP map as it outlines the processes of A\&E; determines object's roles and boundary, as well as level of details; and models events for the discrete-event simulation. The application of STP map in MVSimO, makes various kinds of objects, properties and relations between classes and their instances comprehensible. Beside, STP map act as a bridge for the modeler and the user, and translating the real-world process into an ontological definition. The rationale behind STP in this study is to identify the process elements which can then be applied in the FCA-conceptual exploration to extract suitable concepts, and their taxonomic and non-taxonomic relation.

\section{Process Elements Extraction}

For further development of MVSImO in this study, A\&E data from a London Hospital is used to represent objects and attributes of the domain and by using conceptual exploration approach in FCA, the dependencies between the attributes are described and the concepts are determined. Firstly, FCA formalizes the notion of concept relative to a formal context - objects and attributes mapped into a cross-table. The formalization of concept is based on modeler's perspective on what to model, for the purpose of this: the A\&E pathways. The motivation of concept exploration is to find new relationship for classes in MVSimO, and to have that the focus is given to two objectives; first, to produce a relevant concept and second, to construct the minimal set of implications from the concepts. The followings are the formal definitions of FCA to achieve these two objectives:

- Definition 4.1 Formal context

A formal context is a triplet $(X, Y, I)$ where $X$ is a set of objects and $Y$ is a set of attributes and $I$ is a binary relation between $X$ and $Y$, i.e., $I \subseteq X \times Y$. $(x, y) \in I$ indicates that the object $x$ has attribute $y$.

- Definition 4.2 Intent and Extent

Let $(\mathrm{X}, \mathrm{Y}, \mathrm{I})$ be a context, $\mathrm{X}^{\prime} \subseteq X$ and $\mathrm{Y}^{\prime} \subseteq Y$, the function Intent maps a set of objects to the set of attributes, whereas the function Extent maps a set of attributes to the set of objects:

Intent $\left(\mathrm{X}^{\prime}\right)=\mathrm{y} \in Y^{\prime}-\forall y \in Y^{\prime},(\mathrm{x}, \mathrm{y}) \in R$

Extent $\left(\mathrm{Y}^{\prime}\right)=\mathrm{x} \in X^{\prime}-\forall x \in X^{\prime},(x, y) \in R$ 


\section{Saleh and Bell}

For $\mathrm{X}^{\prime} \subseteq X$, Intent $\left(\mathrm{X}^{\prime}\right)$ is the set of attributes owned by all objects of $\mathrm{X}^{\prime}$, and Extent( $\left.\mathrm{Y}^{\prime}\right)$ is the set of all objects that own the attributes $Y^{\prime}$. The two functions form a Galois connection and formal concepts.

- Definition 4.3 Formal Concept

A Formal Concept $\mathrm{C}$ in a context is a pair $\left(\mathrm{X}^{\prime}, \mathrm{Y}^{\prime}\right)$ that satisfies $\mathrm{Y}^{\prime}=\operatorname{Intent}\left(\mathrm{X}^{\prime}\right)$ and $\mathrm{X}^{\prime}=\operatorname{Extent}\left(\mathrm{Y}^{\prime}\right)$ i.e., $\mathrm{C}$ is a Formal Concept $\Leftrightarrow$ for $\mathrm{X}^{\prime} \in \operatorname{Cand}^{\prime} \in C$, $\operatorname{Extent}\left(\operatorname{Intent}\left(\mathrm{X}^{\prime}\right)\right)=\mathrm{X}^{\prime}$, and symmetrically, Intent $\left(\operatorname{Extent}\left(\mathrm{Y}^{\prime}\right)\right)=\mathrm{Y}^{\prime}$.

- Definition 4.4 Implications

An implication $\mathrm{A} \Rightarrow B$ holds in $(\mathrm{X}, \mathrm{Y}, \mathrm{I})$ if and only if $\mathrm{B} \subseteq A^{\prime \prime}$, which is equivalent to $\mathrm{A}^{\prime} \subseteq B^{\prime}$. It then automatically holds in the set of all concept intents

The attributes, including the transformed single-value data are selected based on processes from STP - Check-In; Assessment; Treatment; and Outcome. These attributes are merely selected according to the processes through A\&E pathways to generate cross-table. The process elements in STP defined as process name (e.g.Outcome), process entity (e.g.Patient), process date and time (e.g.Mon: am), and process location (e.g.Dept: A and E). This step is to obtain concepts with process-related attributes. This process reduced the dataset by only includes the columns with process element hence makes implicit knowledge discovery easier by focusing only on the context of the model, and also makes the representations of FCA concept more process-oriented. The reduced dataset focused on process element by including the attributes of:

Age (Age)

EMAttendanceDate (Attendance Date/Time)

EMModeofArrivalDescription(Mode of Arrival)

AttendanceDisposal (Outcome - Admitted or Discharged)

DepartmentDescription (Department)

ConExp software (Yevtushenko 2000) is used to plot the cross table. ConExp generates implication basis and Concept Lattice diagram to determine any new relationship for MVSimO classes.

\subsection{Implication to Class Relation Translation}

The result from FCA implicitly described the process relation in A\&E department. Using the logical reasoning method by (Xiao Hang Wang, Da Qing Zhang, Tao Gu, and Hung Keng Pung 2004), the implications result from FCA implicitly described the process relation in A\&E department.Considering attribute Age and Attendance Date/Time belonging to Patient class (Subject) in MVSimO, only attributes Mode of Arrival, Admission or Discharge and Department are taken as class properties to describe the process in A\&E. Based on logical reasoning of the first-order predicate - a subject, a verb and an object and referring to the classes in MVSimO, a subject is a class e.g Patient, a verb is a properties-derived implication, and an object is a class e.g Department. As a result from the implications, five concepts with two or more objects are selected as concepts. Table 1 shows the list of formal concepts in natural language statement.

\section{MVSimO Development}

For the development of MVSimO, existing ontology of DeMO is adopted as guideline. An ontology for discrete-event modeling and simulation (DeMO) by (Silver, Miller, Hybinette, Baramidze, and York 2011), provides taxonomies for a discrete-event simulation model that captures the essential features of the real world system. The refinement of MVSimO classes has taken suitable classes from DeMO includes relation properties from conceptual exploration process from previous section. Using method of event scoping and event harmonization from framework by (Bell, De Cesare, Iacovelli, Lycett, and Merico 2007), the first version of MVSimO ontological model is created by deriving the semantic content of the domain. The framework incorporates STP map to replicate the A\&E domain; and the domain knowledge from existing ontology (DeMO). The development is supported by the process relations from Formal Concept Analysis. 
Table 1: Selected Formal Concepts

\begin{tabular}{|l|l|}
\hline Formal concept, expressed in natural language & No.of Object \\
\hline $\begin{array}{l}\text { Fri: pm, MOA:Brought in by Ambulance, Dept: A and E, Age: } 65 \text { and } \\
\text { above, AD:Admitted to hospital bed/LODGED Patient }\end{array}$ & 3 \\
\hline $\begin{array}{l}\text { Thu: pm, MOA:Other, Dept: Mount Vernon MIU, Age: } 65 \text { and above, } \\
\text { AD: Discharged - did not require followup }\end{array}$ & 2 \\
\hline $\begin{array}{l}\text { Sun: pm, MOA:Other, Dept: UCC, Age: 0-4, } \\
\text { AD: Discharged - did not require followup }\end{array}$ & 2 \\
\hline $\begin{array}{l}\text { Mon: am, MOA: Brought in by Ambulance, Dept: A and E, } \\
\text { Age: 19-64, AD: Admitted to hospital/LODGED Patient }\end{array}$ & 2 \\
\hline $\begin{array}{l}\text { Wed: pm, MOA: Other, Dept: mount Vernon MIU, Age: 5-18, } \\
\text { AD: Discharged - did not require followup }\end{array}$ & 2 \\
\hline
\end{tabular}

Incorporating process relations and classes from DeMO enables the detailing of the relationship of the real world knowledge. The artefact can be regenerated to incorporate new ontologies to extract new classes and relationships. This step is necessary to allow for the flexibility of the framework, and to enable the ontology engineer to go back to this step to add new classes and properties and create new ontology. The process of existing ontology adoption in this activity has resulted in the creation of new classes, properties and individuals. Apart from class properties from the existing ontologies, as mentioned earlier, new properties of MVSimO are also derived from the real-world knowledge through the formal concept analysis. Individuals or instances are from the A\&E data. Table 2 summarizes the decisions made and actions taken to elements into MVSimO. Elements are combined to create the initial ontological model.

Table 2: Elements and Class Properties

\begin{tabular}{|l|l|}
\hline Elements & MVSimO Class \\
\hline Process hasOccuranceOf Event & $\begin{array}{l}\text { Process, } \\
\text { new class (Event) }\end{array}$ \\
\hline Patient codedBy Patient ID & $\begin{array}{l}\text { Patient, } \\
\text { new class (Patient ID) }\end{array}$ \\
\hline Patient waitsIn Queue & $\begin{array}{l}\text { Patient, } \\
\text { new class (Queue) }\end{array}$ \\
\hline Queue codedBy Date/Time & $\begin{array}{l}\text { new class (Queue), } \\
\text { new class (Date/Time) }\end{array}$ \\
\hline Event takesPlaceAt Location & $\begin{array}{l}\text { new class (Event) } \\
\text { renamed (Facility) } \\
\text { to (Location) }\end{array}$ \\
\hline Patient servedBy Event & $\begin{array}{l}\text { Patient, } \\
\text { new class (Staff) with } \\
\text { subclass (Nurse) }\end{array}$ \\
\hline Event supportedBy Staff & $\begin{array}{l}\text { new class (Event), } \\
\text { new class (Staff) with } \\
\text { subclass (Nurse) }\end{array}$ \\
\hline
\end{tabular}

In model harmonization activity, the first-cut domain model is combined with DeMO to show the scoped event to be model. To be more specific, event of getPatientCheckIn and getPatientAssessment are presented. This activity uses a process-oriented ontology subclass in DeMO named the ProcessOrientedModel or PIModel. Ontologically, this enables an explicit mapping between real-world DES elements and the domain they serve. The harmonized model is mapped into DeMo PIModel accordingly, and may be translated later to an XML and then into a simulation model. This allows researchers, domain experts and modellers to share a common understanding of the concepts and the relationship of the domain. Harmonized 
model enables an explicit mapping between a process (with its parts), and the domain it serves supported by A\&E data.

Figure 3 presents the harmonization model derived from the event of getPatientCheckIn and getPatientAssessment. The processes are defined within DeMO and their parameter are typed in relation to the respective classes. From the diagram, Process and Patient classes are derived from MinDO; , Location, Event, Staff, Nurse, Queue, Patient ID and Date/Time are new classes or renamed classes after the decisions made on which elements to be taken into MVSimO. Class Check-In, Assessment, Treatment, Outcome, Paramedic and Medical Staff are from Space-Time-Process map, and finally Activity, Entity, Queue, Location, Resources, and Process are mapped-out from DeMO.

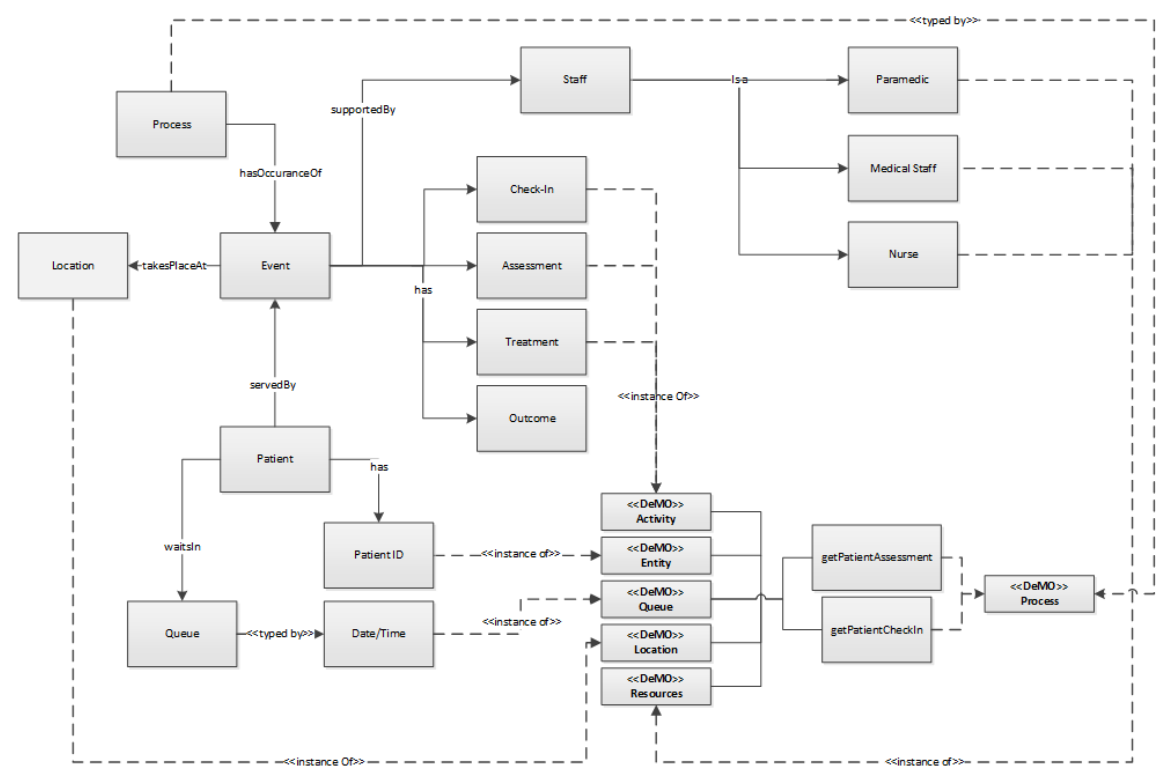

Figure 3: The Harmonized Model

Table 3: MVSimO-Cumberland Objects Assessment

\begin{tabular}{|l|l|l|}
\hline Category & Cumberland Model & MVSimO \\
\hline Data Group & Patient Age & Age \\
\hline & Arrival Method & EMModeofArrivalDescription \\
\hline & Arrival Time & EMAttendanceDate \\
\hline & & DepartmentDescription \\
\hline & & AttendanceDisposal \\
\hline Objects & Departments & Process \\
\hline & Demographic Data & Activity \\
\hline & Resources & Location \\
\hline & & Patient \\
\hline & & Resources \\
\hline & & Queue \\
\hline
\end{tabular}

A comparison of the model presented in this study with existing simulation model, the Cumberland model (Bell, Cordeaux, Stephenson, Dawe, Lacey, and O'Leary 2017) was conducted. The comparison evaluates the functional adequacy and quality of the model by conducting similarity analysis for categories of: Data Group and Objects. Table 3 shows the similarity assessment of the elements in MVSimO and Cumberland model. From element-by-element comparison with 10 elements extracted from MVSimO and 6 elements extracted from Cumberland, 5 of the elements are similar. It can be concluded that $83 \%$ of 


\section{Saleh and Bell}

elements are overlapped and 4 new elements are produced from MVSimO. With a promising comparison result, the process-based ontological model approach presented in this study, a tedious work in collecting domain knowledge and gathering experts' opinions to validate the model can be reduced. The result can also be improved with more elements provided a rigour conceptualization in FCA stage.

\section{Conclusion}

The concept for later version of MVSimO are easily characterized by the modeler or given to adaptation to produce a viable simulation model.Despite these achievements, several issues still need to be addressed in the future by full ontology development, in particular related to more semantic content, considering relations and combining knowledge sources. For future research, the harmonized model of MVSimO can be followed for different concepts of processes to be covered for the development of simulation model in other domain. The development of MVSimO can be revised at every stage to ensure the knowledge obtained by the end of the research is close enough to have a metamodel of A\&E departments. The result of our method heavily depends on A\&E domain where discrete-event simulation is being used to show the sequence of events in the departments. In addition, we are going to explore the possibility to acquire ontology data from different domain and design the ontology to suit other type of simulation like agent-based simulation and system dinamic.

\section{REFERENCES}

Aringhieri, R., M. E. Bruni, S. Khodaparasti, and J. T. van Essen. 2017. "Emergency medical services and beyond: Addressing new challenges through a wide literature review". Computers and Operations Research 78 (August 2016): 349-368.

Baboolal, K., J. D. Griffiths, V. A. Knight, A. V. Nelson, C. Voake, and J. E. Williams. 2012. "How efficient can an emergency unit be a perfect world model". Emergency Medicine Journal 29 (12): 972-977.

Bell, D., C. Cordeaux, T. Stephenson, H. Dawe, P. Lacey, and L. O'Leary. 2017. "Designing effective hybridization for whole system modeling and simulation in healthcare". Proceedings - Winter Simulation Conference:1511-1522.

Bell, D., S. De Cesare, N. Iacovelli, M. Lycett, and A. Merico. 2007. "A framework for deriving semantic web services". Information Systems Frontiers 9 (1): 69-84.

Chahal, K., T. Eldabi, and T. Young. 2013. "A conceptual framework for hybrid system dynamics and discrete event simulation for healthcare". Journal of Enterprise Information Management 26 (1/2): $50-74$.

De Cesare, S., D. Juric, and M. Lycett. 2014. "Toward the automation of business process ontology generation". Proceedings - 16th IEEE Conference on Business Informatics, CBI 2014 1:70-77.

Ghanes, K., M. Wargon, Z. Jemai, O. Jouini, R. Hellmann, V. Thomas, and G. Koole. 2014. "a Comprehensive Simulation Modeling of an Emergency Department: a Case Study for Simulation Optimization of Staffing Levels". Proceedings of the 2014 Winter Simulation Conference:1421-1432.

Grolinger, K., M. A. M. Capretz, J. R. Marti, K. D. Srivastava, K. Grolinger, and M. A. M. Capretz. 2012. "Ontology - based Representation of Simulation Models Ontology - based Representation of Simulation Models". The Twenty-Fourth International Conference on Software Engineering and Knowledge Engineering (SEKE):432-437.

Gruber, T. R. 1993. "Toward Principles for the Design of Ontologies Used for Knowledge Sharing. KSL 93-04". Knowledge Systems Laboratory, Stanford University.

Guizzardi, G., and G. Wagner. 2010. "Towards an ontological foundation of discrete event simulation". Proceedings - Winter Simulation Conference:652-664.

Gul, M., and A. F. Guneri. 2015. "A comprehensive review of emergency department simulation applications for normal and disaster conditions". Computers and Industrial Engineering 83:327-344. 


\section{Saleh and Bell}

Günal, M. M., and M. Pidd. 2009. "Understanding target-driven action in emergency department performance using simulation.". Emergency medicine journal : EMJ 26:724-727.

Huang, Y.-L. 2016. "Appointment standardization evaluation in a primary care facility". International Journal of Health Care Quality Assurance 29 (6): 675-686.

Isern, D., and A. Moreno. 2016. "A Systematic Literature Review of Agents Applied in Healthcare". Journal of Medical Systems 40 (2): 1-14.

Lebcir, R., E. Demir, R. Ahmad, C. Vasilakis, and D. Southern. 2017. "A discrete event simulation model to evaluate the use of community services in the treatment of patients with Parkinson's disease in the United Kingdom". BMC Health Serv Res 17 (1): 50.

Lonsdale, D., D. W. Embley, Y. Ding, L. Xu, and M. Hepp. 2010. "Reusing ontologies and language components for ontology generation". Data and Knowledge Engineering.

Malone, J., R. Stevens, S. Jupp, T. Hancocks, H. Parkinson, and C. Brooksbank. 2016. "Ten Simple Rules for Selecting a Bio-ontology". PLoS Computational Biology 12 (2): 1-6.

Noy, Natalya F and McGuinness, Deborah L and others 2001. "Ontology development 101: A guide to creating your first ontology".

Pidd, M. 2014. "The ways forward : A personal view of system dynamics and discrete-event simulation". Discrete-Event Simulation and System Dynamics for Management Decision Making:318-336.

Ries, E. 2009. "Minimum Viable Product: a guide". Startup Lessons Learned.

Robinson, S. 2013. "Conceptual modeling for simulation". 2013 Winter Simulations Conference (WSC):377388.

Sider, T. et al. 2001. Four-dimensionalism: An ontology of persistence and time. Oxford University Press on Demand.

Silver, G. A., J. A. Miller, M. Hybinette, G. Baramidze, and W. S. York. 2011. "DeMO: An Ontology for Discrete-event Modeling and Simulation". Simulation 87 (9): 747-773.

Simperl, E. 2009. "Reusing ontologies on the Semantic Web: A feasibility study". Data \& Knowledge Engineering 68 (10): 905-925.

Stachowiak, H. 1973. Allgemeine modelltheorie. Springer.

Traore, M. K., G. Z. R. D., and B. Zeigler. 2018. "Modeling and simulation framework for value-based healthcare systems".

Wille, R. 1982. "Restructuring Lattice Theory: An Approach Based on Hierarchies of Concepts". In Ordered Sets: Proceedings of the NATO Advanced Study Institute held at Banff, Canada, August 28 to September 12, 1981, edited by I. Rival, 445-470. Dordrecht: Springer Netherlands.

Xiao Hang Wang, Da Qing Zhang, Tao Gu, and Hung Keng Pung. 2004. "Ontology based context modeling and reasoning using OWL". IEEE Annual Conference on Pervasive Computing and Communications Workshops, 2004. Proceedings of the Second:18-22.

Yevtushenko, S. 2000. "Documentation - The Concept Explorer". Proceedings of the 7th national conference on Artificial Intelligence:127-134.

\section{AUTHOR BIOGRAPHIES}

NURUL SALEH received a BSc (Hons) Information System from Multimedia University, Malaysia and obtained her Master's Degree in Software Engineering from Universiti Putra Malaysia. She worked in industrial sectors before completed her $\mathrm{PhD}$ in Computer Science at Brunel University in 2019. She is currently a researcher in the Department of Computer Science at Brunel University.

DAVID BELL is a reader in the Department of Computer Science at Brunel University where he teaches and carries out research into Software Services, Cybersecurity and Modelling \& Simulation. Before returning to university, he worked for several small and large software development companies and then in Investment Banking becoming technology director leading Emerging Markets and Trade Capture initiatives globally. 